\title{
Prácticas de liderazgo de docentes y directivos que favorecen el aprendizaje de estudiantes en contexto mapuche
}

Leadership practices of teachers and managers

that favor student learning in the mapuche context

María Elena Mellado Hernández ${ }^{\mathrm{a}}$ \& Juan Carlos Chaucono Catrinao $^{\mathrm{b}}$

aFacultad de Educación, Universidad Católica de Temuco, Chile, $\$ mmellado@uct.cl [orcid.org/0000-0001-8358-0845]

bFacultad de Educación, Universidad Católica de Temuco, Chile, juancarloschauconocatrinao@gmail.com [orcid.org/0000-0002-6021-7047]

\section{RESUMEN}

El objetivo de esta investigación consiste en indagar las prácticas de liderazgo de docentes y directivos que facilitan el aprendizaje de estudiantes de una escuela primaria en contexto rural mapuche, región de La Araucanía, Chile. Se planteó un diseño cualitativo suscrito a un estudio de caso. Los resultados develan prácticas de liderazgo centradas en la mejora de los aprendizajes desde una perspectiva intercultural, basadas principalmente en una relación constante entre el conocimiento mapuche y el conocimiento escolar, lo que nutre el desarrollo de un trabajo colaborativo, decisiones compartidas y acciones contextualizadas y significativas que favorecen el mejoramiento del aprendizaje de los estudiantes.

PALABRAS CLAVE: prácticas liderazgo intercultural, aprendizajes, estudiante, comunidad mapuche.

\section{ABSTRACT}

The objective of this research is to investigate the leadership practices of teachers and managers that facilitate the learning of students of an elementary school in rural Mapuche context, region of La Araucanía, Chile. A qualitative design was subscribed to a case study. The results reveal leadership practices focused on improving learning from an intercultural perspective, based mainly on a constant relationship between Mapuche 
knowledge and school knowledge, which nurtures the development of collaborative work, shared decisions and contextualized and meaningful actions that favor the improvement of student learning.

KEY WORDS: intercultural leadership practices, learning, student, Mapuche community.

\section{INTRODUCCIÓN}

Liderar los centros escolares en contexto rural mapuche desde una perspectiva monocultural occidental se ha transformado en una de las problemáticas no resueltas en Chile que atenta con el desarrollo de buenos aprendizajes en niños, niñas y jóvenes que asisten a estas escuelas (Quintriqueo \& Torres, 2013; Mellado \& Chaucono, 2016; Beltrán \& Osses, 2018). Al respecto, diversas investigaciones han constatado que a pesar de los avances investigativos sobre educación intercultural se conservan prácticas pedagógicas y directivas descontextualizadas, centradas en la enseñanza del profesorado, obviando contexto y características del estudiante (Lara, 2014; Quintriqueo, Morales, Quilaqueo \& Arias, 2016; Chaucono, Mellado \& Yuste, 2017). De esta manera, se espera que tanto docentes como directivos mejoren los procesos pedagógicos en virtud de la diversidad étnica y cultural de la población escolar que atienden.

En relación a lo anterior, las condiciones que caracterizan a la educación rural en términos de ubicación geográfica, población que atiende y singularidades pedagógicas ameritan una postura educativa diferente que considere los intereses, necesidades y características de la comunidad educativa con el propósito de introducir estos saberes identitarios al currículum educativo, creando oportunidades que permitan mejorar la calidad de los aprendizajes. Al respecto, diversos estudios manifiestan que construir procesos educativos desde una relación con el territorio rural requiere de una amplia participación de todos los actores, de una descentralización curricular y de un enfoque pedagógico flexible centrado en el estudiante (Rojas, 2013; Mellado \& Huaiquimil, 2015; Chaucono \& Mellado, 2017; Tinajero \& Solís, 2019). Todo lo anterior, se propone desde el desarrollo de un liderazgo compartido, de aprender de manera conjunta, de decisiones colectivas y de responsabilidades mutuas en pro del mejoramiento escolar.

De esta manera, el liderazgo escolar se expresa como un conjunto de prácticas horizontales que surgen desde la colaboración entre los distintos integrantes de la comunidad educativa. En esta misma línea, los estudios sobre liderazgo en comunidades indígenas manifiestan que gestionar la participación comunitaria fortalece el desarrollo territorial local, transformándolo en un escenario pedagógico que propicia la construcción de aprendizajes contextualizados y pertinentes (Rojas, 2013; Buitrago, Baptista \& Chiquinquirá, 2014; Arias, 2017; Gutiérrez, Martín \& Jenaro, 2018). Del mismo modo, Beltrán \& Pérez (2018) manifiestan que los equipos directivos que se desempeñan en 
contexto intercultural debiesen promover actividades de aprendizaje que alienten el trabajo colaborativo entre todos los integrantes de la comunidad. En otras palabras, los líderes escolares en contexto rural mapuche debiesen transformar las escuelas en comunidades de aprendizaje con el fin de construir una relación formadora entre el conocimiento mapuche y el conocimiento escolar.

Lo anterior, exige ampliar la mirada hacia la construcción de aprendizajes desde el contexto sociocultural del estudiante. De este modo, desarrollar prácticas de liderazgo pedagógico en contexto mapuche requiere de reflexiones (rakizuam)democráticas, donde las conversaciones entre los integrantes de la comunidad mapuche y escolar son oportunidades para formar al otro a través del análisis del contexto cultural y social, de los intereses y las necesidades de formación educativa de los pichikiche (niños y niñas) que demanda la comunidad, pero dialogado desde una mirada intercultural con el fin de construir entre todos una enseñanza auténtica y significativa para los niños y niñas de la escuela. Siguiendo este marco, y desde una perspectiva occidental, diversos autores sostienen que cuando los líderes educativos asumen su responsabilidad en formar al profesorado desde las necesidades y problemáticas que surgen desde la sala de clases, impactan indirectamente en el aprendizaje del estudiante (Bellei, Valenzuela, Vanni \& Contreras, 2014; Bellei, Valenzuela, Vanni \& Contreras, 2014; Robinson, Lloyd \& Rowe, 2014; Bolívar, 2015; Murillo \& Krichesky, 2015; Day, Gu \& Sammons, 2016; Bolívar, Caballero \& García, 2017; Bender, 2017; Fullan, 2017; Mellado, Chaucono \& Villagra, 2017; Weinstein \& Muñoz, 2017; Rincón Gallardo, 2019). De este modo, orientar los procesos de mejora escolar desde los conocimientos y saberes cotidianos del contexto cultural optimiza la pertinencia de la enseñanza y garantiza para el estudiante verdaderas oportunidades de aprender.

En este contexto, las prácticas de liderazgo escolar son aquellas acciones educativas desarrolladas por el equipo directivo que influyen en la motivación y en las habilidades de la enseñanza de los docentes, preocupándose de crear condiciones favorables para asegurar que todo el estudiantado aprenda en contexto diverso. En este sentido, distintos autores (Leithwood, Day, Sammons, Harris \& Hopkins, 2006; Leithwood, 2011; Sammons, Gu, Day \& Ko, 2011), han identificado cuatro tipos de prácticas de liderazgo que tienen impacto positivo en el aprendizaje del estudiante. Éstas se describen en la Tabla 1.

De este modo, se establecen 4 prácticas directivas que potencian el desarrollo de un sistema de mejoramiento continuo cuyo núcleo central es sostener las condiciones organizacionales e impactar positivamente en el proceso de aprender del estudiante. En Chile, el Ministerio de Educación (2015) establece en el Marco para la Buena Dirección y Liderazgo Escolar (MBD y LE) cómo un conjunto de prácticas de liderazgo impulsadas por habilidades, conocimientos y actitudes son necesarias para gestionar y liderar una organización educativa. Dicho marco considera cinco dimensiones de prácticas: Construyendo e implementando una visión estratégica compartida, Desarrollando las capacidades profesionales, Liderando los procesos de enseñanza y aprendizaje, Gestionando la convivencia y la participación de 
Tabla 1. Prácticas del liderazgo escolar que impactan en el aprendizaje del estudiante.

\begin{tabular}{|c|c|}
\hline Práctica & Descripción \\
\hline Establecer una dirección & $\begin{array}{l}\text { Los directores efectivos proveen de una visión clara y un sentido a la escuela, } \\
\text { desarrollando una comprensión compartida y misión común de la organización, } \\
\text { focalizada en el progreso del estudiantado. Para esto desarrollan prácticas tales } \\
\text { como: identifica nuevas oportunidades para la organización motivando e } \\
\text { incentivando al personal para conseguir las metas comunes. Esto implica establecer } \\
\text { valores al staff y a los estudiantes de acuerdo a las alianzas. }\end{array}$ \\
\hline Desarrollar al personal & $\begin{array}{l}\text { Habilidad del líder para potenciar aquellas capacidades de los miembros de la } \\
\text { organización necesarias para movilizarse de manera productiva en función de dichas } \\
\text { metas. Prácticas coherentes son: desarrollo profesional, atención, incentivos o } \\
\text { apoyos, procesos deliberativos que amplien la capacidad de los miembros para } \\
\text { responder mejor a los desafios. }\end{array}$ \\
\hline Rediseñar la organización & $\begin{array}{l}\text { Establecer condiciones de trabajo que posibiliten al personal un desarrollo de sus } \\
\text { motivaciones y capacidades con prácticas que construyan una cultura colaborativa, } \\
\text { faciliten el trabajo en equipo y gestionen el entorno de trabajo. Para eso, se han de } \\
\text { posibilitar la creación de tiempos comunes de planificación para profesores, } \\
\text { estableciendo estructuras grupales para la resolución de problemas, distribución del } \\
\text { liderazgo y mayor implicación del profesorado en la toma de decisiones. }\end{array}$ \\
\hline $\begin{array}{c}\text { Gestionar los programas de enseñanza y } \\
\text { aprendizaje }\end{array}$ & $\begin{array}{l}\text { Conjunto de tareas destinadas a supervisar y evaluar la enseñanza, coordinar el } \\
\text { currículum, proveer los recursos necesarios y seguir el progreso de los estudiantes. } \\
\text { Prácticas adecuadas son: supervisar la sala de aula, motivar emocionalmente al } \\
\text { profesorado con actitud de confianza hacia ellos y sus capacidades promoviendo su } \\
\text { iniciativa y apertura a nuevas ideas y prácticas. }\end{array}$ \\
\hline
\end{tabular}

Fuente: Elaboración adaptada del Centro de Estudios de Políticas y Prácticas en Educación (CEPPE) en base a Leithwood et al. (2006).

la comunidad escolar y Desarrollando y gestionando el establecimiento escolar. En relación a lo anterior, la Tabla 2 describe las prácticas de liderazgo abordadas en este estudio, contextualizadas a una comunidad rural mapuche.

Por consiguiente, surge la necesidad de indagar en las prácticas de liderazgo que facilitan el aprendizaje del estudiante en una escuela en contexto rural mapuche, región de La Araucanía, Chile.

\section{METODOLOGÍA}

La investigación corresponde a un estudio de caso que utiliza una metodología cualitativa con un enfoque participativo cuyo propósito es construir conocimiento colectivo entre todos los agentes participantes para iniciar procesos que aporten a la mejora educativa en sus propias comunidades (Guba \& Lincoln, 2012).

\section{Participantes}

En este estudio de caso participan 8 personas que corresponden a toda la comunidad educativa que atiende a estudiantes en una escuela rural mapuche de la región de La Araucanía, Chile. La selección de los participantes fue intencional y según Gurdián (2007) afirma que 
Tabla 2. Descripción de prácticas de liderazgo escolar contextualizadas al contexto mapuche.

\begin{tabular}{|c|c|}
\hline Prácticas de liderazgo & Descripción \\
\hline $\begin{array}{l}\text { Construyendo una visión estratégica } \\
\text { compartida }\end{array}$ & $\begin{array}{l}\text { Las prácticas de liderazgo en la dimensión construyendo una visión } \\
\text { estratégica compartida tributan a gestar de manera participativa entre } \\
\text { todos los integrantes de la comunidad un lenguaje común sobre como se } \\
\text { enseña, aprende y evalúa en contexto intercultural. Al respecto, Beltrán } \\
\text { y Osses (2018) declaran que promover la participación comunitaria en la } \\
\text { construcción de una mirada común favorece el reencuentro de las } \\
\text { distintas cosmovisiones. En otras palabras, construir una visión } \\
\text { compartida en escuelas rurales indígenas requiere del desarrollo de } \\
\text { prácticas de liderazgo que promocionen la participación de todos los } \\
\text { integrantes que componen la comunidad. }\end{array}$ \\
\hline Desarrollando capacidades profesionales & $\begin{array}{l}\text { Las prácticas de liderazgo en la dimensión desarrollando capacidades } \\
\text { profesionales se focalizan en el conjunto de actividades que debiese } \\
\text { realizar el profesorado como resultado de las interacciones significativas } \\
\text { y de los espacios de reflexión crítica abordados en el centro escolar, } \\
\text { dando lugar a cambios en la práctica de aula y en los modos de pensar } \\
\text { dicha práctica. En concordancia con lo anterior, Vaillant y Marcelo (2015) } \\
\text { afirman que el mejoramiento de la práctica pedagógica forma parte de } \\
\text { una construcción de aprendizaje colectiva nacidas desde el análisis y } \\
\text { cuestionamientos de los saberes previos de la enseñanza. En otras } \\
\text { palabras, las propias necesidades de aprendizaje profesional tanto de } \\
\text { docentes como de directivos emergen en las discusiones profesionales } \\
\text { como en la propia práctica pedagógica vinculadas directamente en el } \\
\text { cómo se enseña, aprende y evalúa en el contexto rural indígena. }\end{array}$ \\
\hline $\begin{array}{l}\text { Liderando los procesos de enseñanza } \\
\text { aprendizaje }\end{array}$ & $\begin{array}{l}\text { Las prácticas de liderazgo en la dimensión liderando los procesos de } \\
\text { enseñanza y aprendizaje se vinculan directamente con la forma como los } \\
\text { directivos y docentes conciben buenas prácticas de aprendizaje en } \\
\text { relación a su contexto sociocultural y características de los estudiantes. } \\
\text { De esta manera, Mellado y Chaucono (2015) manifiestan que crear } \\
\text { oportunidades de aprendizaje significativas y pertinentes al contexto } \\
\text { intercultural requiere de un dominio disciplinar, pedagógico y cultural. } \\
\text { De este modo, desarrollar prácticas de liderazgo en contexto rural } \\
\text { indigena requiere de una formación participativa y recíproca entre los } \\
\text { conocimientos mapuche y escolares. }\end{array}$ \\
\hline $\begin{array}{l}\text { Gestionando la participación de la } \\
\text { comunidad escolar }\end{array}$ & $\begin{array}{l}\text { Las prácticas de liderazgo en la dimensión gestionando la participación } \\
\text { de la comunidad escolar se centran en promover un clima basado en } \\
\text { relaciones de confianza que aseguren la inclusión de todos los } \\
\text { integrantes de la comunidad. En este sentido, Murillo \& Krichesky (2015) } \\
\text { sostienen que gestionar la participación comunitaria provoca relaciones } \\
\text { de confianza en pro de asegurar un trabajo colaborativo que propicie el } \\
\text { aprendizaje de todos. Lo anterior, permite desarrollar de manera } \\
\text { participativa y democrática prácticas para el logro de una sana } \\
\text { convivencia entre todos los integrantes de la comunidad escolar. }\end{array}$ \\
\hline Desarrollando el establecimiento escolar & $\begin{array}{l}\text { Las prácticas de liderazgo en la dimensión desarrollando el } \\
\text { establecimiento escolar, se relacionan principalmente con la necesidad } \\
\text { de transformar la organización educativa en virtud de la recolección y } \\
\text { análisis de la información y datos de los procesos y resultados del } \\
\text { establecimiento con el fin de tomar decisiones pertinentes y adecuadas a } \\
\text { la realidad local. Siguiendo este marco, Chaucono, Mellado y Yuste } \\
\text { (2017) argumentan que considerar las necesidades del territorio local } \\
\text { garantiza entornos que contribuyan a la mejora de la comunidad escolar } \\
\text { en su conjunto. }\end{array}$ \\
\hline
\end{tabular}

Fuente: Elaboración propia de los autores en base al M.B.D. y L.E. (2015). 
para seleccionar a los sujetos se requiere elegir a quienes tengan un mejor conocimiento del constructo a investigar, de este modo se garantiza una saturación efectiva de la información a categorizar. Sus edades fluctúan entre 35 y 63 años de edad, de los cuales cinco son mujeres de ascendencia mapuche, tres de ellas poseen título de profesora de Educación General Básica y dos pertenecen a la comunidad mapuche, asumiendo en este contexto roles de consejeras pedagógicas. En relación a la cantidad de hombres, éstos corresponden a tres, uno de ellos tiene formación en Liderazgo Escolar desempeñándose como director del centro educativo y dos de ellos, mención en Lenguaje y Comunicación. Respecto a los años de experiencia como docente rural cuatro de ellos tienen entre 8 y 11 años y el resto, más de 20 años desempeñándose como docente rural.

\section{Técnica e instrumentos de recogida de datos}

En este estudio para la recogida de información se utilizaron las técnicas de grupos de discusión y observación participante.

En primer lugar, se diseña para el grupo de discusión un guión de preguntas orientadoras con el fin de propiciar el diálogo que permita indagar en las prácticas directivas que facilitan la mejora de los aprendizajes del estudiantado. En este caso, se levantó una pregunta para cada dimensión del MBD y LE, validadas por jueces expertos. A continuación, la Tabla 3 presenta la estructura del guión de preguntas orientadoras.

Posteriormente, se procede a realizar tres grupos de discusión con la totalidad de los participantes en un periodo de seis meses con una duración de 60 minutos, lo que fue registrado por medio de audio previo consentimiento informado. En el desarrollo de la conversación surgió una instancia de reflexión (rakizuam) colectiva, en la que se visualizó los significados que le atribuyen a las prácticas de liderazgo escolar los participantes que asumen distintos roles en el centro escolar.

Tabla 3. Estructura del guión de preguntas orientadoras para el grupo de discusión.

\begin{tabular}{|c|c|}
\hline Dimensiones de prácticas de liderazgo escolar & Preguntas orientadoras \\
\hline $\begin{array}{l}\text { Construyendo e implementando una visión } \\
\text { estratégica compartida }\end{array}$ & $\begin{array}{l}\text { ¿De qué forma se construye una visión } \\
\text { compartida en una escuela en contexto } \\
\text { mapuche? }\end{array}$ \\
\hline Desarrollando las capacidades profesionales & $\begin{array}{l}\text { ¿Dé qué manera se desarrollan las capacidades } \\
\text { profesionales en una escuela en contexto } \\
\text { mapuche? }\end{array}$ \\
\hline $\begin{array}{l}\text { Liderando los procesos de enseñanza y } \\
\text { aprendizaje }\end{array}$ & $\begin{array}{l}\text { ¿En qué sentido las prácticas de liderazgo se } \\
\text { focalizan en los procesos de enseñanza del } \\
\text { profesorado y aprendizaje del estudiante? }\end{array}$ \\
\hline $\begin{array}{l}\text { Gestionando la convivencia y la participación de } \\
\text { la comunidad escolar }\end{array}$ & $\begin{array}{l}\text { ¿Cómo se gestiona y lidera un establecimiento } \\
\text { escolar en contexto mapuche? }\end{array}$ \\
\hline $\begin{array}{l}\text { Desarrollando y gestionando el establecimiento } \\
\text { escolar }\end{array}$ & $\begin{array}{l}\text { ¿Cómo se construye una escuela intercultural } \\
\text { mapuche? }\end{array}$ \\
\hline
\end{tabular}

Fuente: Elaboración propia. 
En segundo lugar, se utilizó la observación participante, la información se obtuvo por medio de registros etnográficos y de audio de las prácticas de liderazgo desarrolladas en las jornadas de reuniones de docentes - directivo. Se realizaron análisis de contenido de las prácticas focalizadas en las dimensiones. En total se registraron durante un año académico 20 sesiones de prácticas directivas distribuidas en 120 minutos con el propósito de contribuir al mejoramiento escolar desde la construcción del conocimiento colectivo y contextualizado.

\section{Análisis de la información}

La información se transcribió en documento formato word, para luego analizar los discursos que resultaron ser recurrentes, esto permitió levantar categorías de prácticas de liderazgo centrado en el aprendizaje del estudiante. En una primera fase, se describen las prácticas de liderazgo pedagógico, luego se muestran los hallazgos a toda la comunidad educativa con el propósito de profundizar en las descripciones desde el análisis de los participantes construyendo desde ahí un conocimiento colectivo, contextualizado y pertinente a la realidad sociocultural. En una segunda fase, se dio paso al proceso de validación de las descripciones de las prácticas, a través del diálogo reflexivo entre los participantes se evaluó la congruencia referida a la concordancia existente entre la descripción de la práctica y su realización en el centro escolar y la coherencia asociada a la relación entre la descripción y la práctica. De este modo, la validación de interesados aportó a través del criterio de praxis, que se garantizará la legitimidad de asegurar que las prácticas sean validadas desde y con la comunidad en base a las experiencias vividas como integrantes que participan activamente de la comunidad educativa. Posteriormente, se procede analizar la información recogida en el registro etnográfico previa ampliación de los datos con el audio de las reuniones con el propósito de levantar de manera ascendente el ciclo de mejoramiento de las prácticas de liderazgo pedagógico centradas en el aprendizaje del estudiante. Finalmente, se presentan los resultados por dimensión y categoría ejemplificando con citas textuales las comprensiones que atribuyen la comunidad a las prácticas de liderazgo escolar. El sistema utilizado para identificar las textualidades se ha organizado de la siguiente manera: Docente, se utiliza la sigla D 1 con numeración secuenciada hasta D 5, consejeras pedagógicas mapuche con la sigla Cpm desde Cpm1 hasta Cpm2 y Directivo, se registra con la sigla DI 1.

\section{RESULTADOS}

En este apartado se presentan los resultados en virtud de los objetivos del estudio y desde el análisis de contenido de las dimensiones analizadas.

En relación a la dimensión "Construyendo e implementando una visión estratégica compartida”, surge la primera categoría. 
Categoría 1: Práctica de liderazgo que involucra a la comunidad mapuche en las decisiones pedagógicas y directivas

Esta categoría hace referencia a las prácticas de liderazgo escolar que desarrollan los docentes y directivos para construir una visión compartida de escuela en contexto mapuche. Éstas se describen como acciones formadoras que se intencionan en las reuniones de apoderados para promover la participación de todos los actores. A continuación, se mencionan algunas respuestas que resultaron ser frecuentes en relación a la pregunta:

¿De qué forma se construye una visión compartida en una escuela en contexto mapuche?

"Yo creo que las reuniones de apoderados que realiza el director junto a los docentes pueden formar una mirada compartida de escuela mapuche, porque en ella reflexionamos todos sobre las acciones que se están realizando para que nuestros pichikona [hijos e hijas]aprendan, lo que ha generado reuniones más participativas y un mayor involucramiento de los peñis [hermano mapuche] (Cpm2).

"Para mí como profesor mapuche fue cuando la escuela abrió sus puertas a la comunidad, en ese momento hubo un cambio, empezaron a involucrarse de manera más representativa a través de sus saberes ancestrales, lo que ha permitido mejorar las relaciones con los apoderados desde una mirada común de lo que entendemos por formación educativa en nuestro contexto" (D 5).

"La escuela empezó a construir una visión compartida cuando empezó a considerar en las clases la realidad local, los intereses y la forma que tenemos como personas mapuche y no mapuche de aprender" (D 4).

“Construir una visión compartida en una escuela en contexto mapuche requiere de prácticas de liderazgo comunitario, de acciones conjuntas y por sobre todo de una vinculación constante entre objetivo de aprendizaje, intereses de la comunidad mapuche, contexto sociocultural y conocimiento mapuche" (DI 1).

En los registros anteriores, se logra visualizar que las prácticas de liderazgo pedagógico centradas en construir un lenguaje común de escuela mapuche se realizan desde el contexto sociocultural del estudiante, considerando de esta manera los intereses, las particularidades y los saberes ancestrales propios de la comunidad mapuche. Desde esta perspectiva intercultural, se asume un liderazgo escolar más participativo e inclusivo. Del mismo modo, se evidencia que descentralizar la gestión para la toma de decisiones favorece las 
relaciones de confianza y las responsabilidades mutuas en aras de construir desde adentro una visión compartida de escuela mapuche. Al respecto, Quintriqueo et al. (2016) afirman que considerar a la comunidad mapuche en la gestión formativa de sus hijos propicia el desarrollo de prácticas comunitarias que permitan mejorar la construcción de aprendizajes contextualizados, separando de esta manera las inequidades entre mapuche y no mapuche.

Respecto a la dimensión "Desarrollando las capacidades profesionales" aflora la segunda categoría.

Categoría 2: Práctica de liderazgo centrada en la reflexión - acción del quehacer docente directivo

Esta categoría hace alusión a la práctica de liderazgo pedagógico que desarrollan directivos y docentes en una escuela mapuche, ésta se caracteriza por centrar la reflexión en la mejora de las actividades de aprendizaje del estudiante, a través de acciones compartidas que exigen de parte del profesorado y líder escolar un dominio curricular, pedagógico y cultural. A continuación, se mencionan algunas respuestas que resultaron ser recurrentes frente a la pregunta:

¿De qué manera se desarrollan las capacidades profesionales en una escuela en contexto mapuche?

"Las prácticas para desarrollar las capacidades profesionales que realizamos en la escuela siempre son reflexionadas por todos y los acuerdos se transforman en acciones que apuntan a que los estudiantes mejoren sus aprendizajes, pero desde una mirada cultural mapuche" (D 1).

"Yo creo que analizar los resultados académicos es una de las oportunidades para mejorar las capacidades profesionales de nosotros como docentes, ya que el propósito siempre es identificar los aprendizajes que requieren ser mejorados o abordados en profundidad para que el estudiante aprenda con sentido y pertinencia" (D 3).

"Desarrollar capacidades profesionales en la escuela en contexto mapuche requiere de acciones conjuntas entre docentes y directivos para diseñar de manera colaborativa la clase, lo que significa que entre todos y todas mejoremos las actividades de aprendizajes desde el contexto sociocultural del estudiante" (DI 1).

"Nosotros junto con los colegas en reuniones mensuales realizamos un seguimiento de los niveles de aprendizaje que han desarrollado los estudiantes. Considero que esta 
acción apunta al desarrollo de capacidades profesionales docentes" (D 2).

"Para mí, desarrollar las capacidades profesionales comienza cuando nos juntamos con los colegas a trabajar colaborativamente sobre la comprensión del currículum escolar y los conocimientos mapuche previos que ha construido el estudiante en su experiencia de vida, todo esto con el fin de realizar un mapeo curricular que identifique y aclare la progresión de los aprendizajes que requieren desarrollar los estudiantes en cada asignatura" (D 4).

El profesorado y equipo directivo, en su mayoría, manifiestan argumentos que dan cuenta que las prácticas de liderazgo pedagógico que permiten desarrollar las capacidades profesionales en una escuela mapuche tributan principalmente al desarrollo de un trabajo colaborativo centrado en comprender el currículum escolar desde el conocimiento mapuche, lo que demanda una mirada reflexiva focalizada en el aprender del estudiante en contexto intercultural. En este sentido, Mellado \& Huaiquimil (2015) manifiestan que el saber pedagógico e intercultural permite desarrollar verdaderos escenarios de aprendizajes contextualizados y de activa participación del estudiante. Asimismo, distintos estudios (Robinson et al. 2014; Bender, 2017) manifiestan que facilitar un contexto favorable y un lenguaje común sobre lo que significa aprender brinda oportunidades para liderar la formación docente y el desarrollo integral del estudiante. De este modo, se testifican que las prácticas de liderazgo pedagógico se nutren sistemáticamente en mejorar la calidad de la enseñanza que realiza el profesorado y en la mejora de los aprendizajes del estudiantado en virtud de los saberes culturales de los niños y niñas.

En la dimensión "Liderando los procesos de enseñanza y aprendizaje”, surgió la tercera categoría:

Categoría 3: Práctica de liderazgo focalizada en el seguimiento del aprendizaje del estudiante

En la mayoría de los discursos derivados en los grupos de discusión realizados a docentes y directivos aparecen recurrentemente argumentos a favor de las experiencias previas que trae consigo el estudiante mapuche, lo cual es valorado positivamente como una oportunidad para vincularlos con los conocimientos, habilidades y actitudes emanados desde las bases curriculares de educación básica. De manera específica, se presentan algunos argumentos que fueron frecuentes frente a la pregunta:

¿En qué sentido las prácticas de liderazgo se focalizan en los procesos de enseñanza del profesorado y aprendizaje del estudiante? 
"A través de la discusión profesional teniendo como foco mejorar nuestras propias prácticas pedagógicas, mediante el desarrollo de actividades de aprendizajes que consideren las experiencias previas que trae el estudiante" (D 2).

"Mediante el proceso de diseñar las clases junto a otros colegas considerando los intereses, las particularidades culturales, las necesidades y los desafíos que debemos desarrollar en los niños y niñas" (D 3).

"Con el trabajo colaborativo que realizamos en clases con los colegas, usando de referente los indicadores de aprendizaje construidos con los estudiantes, lo que permite identificar las buenas prácticas pedagógicas que realizamos en la escuela en atención a los intereses de los niños y niñas" (D 1).

"Siempre nos preguntan en la escuela si podríamos dar a conocer algunos de los relatos mapuche (epew o piam) de la comunidad e incluso el director nos ha pedido que le enseñemos a él y a los profesores, la forma de abordar desde el rakizuam (reflexión) para trabajarlos en las clases y también en las reuniones de apoderados" (Cpm1).

"Con la identificación de las dificultades de aprendizaje presentadas en clases por el estudiante con el propósito de elaborar acciones de mejora que permitan profundizar y vincular los conocimientos mapuche con los conocimientos, habilidades y actitudes que sugieren las bases curriculares del Ministerio de Educación de Chile" (DI 1).

Los extractos de los grupos de discusión permiten testificar que las prácticas de liderazgo focalizadas en los procesos de enseñanza aprendizaje están vinculadas directamente con el desarrollo de un aprendizaje integral, que atiende los conocimientos y saberes culturales de la comunidad mapuche. De esta manera, se destaca la discusión profesional y el rakizuam (reflexión) como una oportunidad para indagar los saberes previos del estudiante y cuestionar las propias prácticas con el fin de mejorar las situaciones de aprendizaje del estudiante. Al respecto, Fullan (2017) \& Rincón Gallardo (2019) afirman la necesidad de vincular los procesos de enseñanza con la formación profesional, lo que requiere de una conexión profunda de los modos de pensar la enseñanza y el aprendizaje. En este sentido, se observan prácticas de liderazgo escolar que logran resignificar el quehacer rutinario en los modos habituales que se tienen de enseñar, aprender y evaluar en contexto intercultural.

En la dimensión "Gestionando la convivencia y la participación de la comunidad escolar" brotó la cuarta categoría: 
Categoría 4: Práctica de liderazgo que propicia el aprendizaje integral de todos

La mayoría de los argumentos planteados por la comunidad escolar explica que la práctica de liderazgo pedagógico de escuela en contexto mapuche se centra recurrentemente en promover un trabajo colaborativo desde la participación activa de integrantes que conforman la escuela, propiciando el aprendizaje integral de todos. En específico se muestran algunos testimonios que documentan lo descrito anteriormente, frente a la pregunta:

¿Cómo se gestiona y lidera un establecimiento escolar en contexto mapuche?

"Las prácticas que desarrollamos en la escuela tributan en su mayoría a valorar las creencias, costumbres y conocimientos del pueblo mapuche, transformándolo en una oportunidad para construir ambientes de confianza y respeto mutuo entre todos los integrantes que conforman la comunidad educativa tanto mapuche como no mapuche" (DI 1).

"Nosotros como docentes construimos desde los distintos espacios de participación, como son las actividades de aprendizaje en clases, las reuniones de apoderados y los relatos que nos ofrecen los sabios mapuche de la comunidad como encuentros formativos que nos permiten asegurar el desarrollo de una sana convivencia escolar en los distintos escenarios educativos" (D 4).

"Para mí como apoderada e integrante de la escuela, la mayoría de las prácticas que realizamos se focalizan en desarrollar competencias que nos permitan valorar puntos de vista distintos, escuchar de manera atenta las opiniones de otras personas no mapuche y autorregularse cuando les quiero decir wuinka (persona no mapuche)" (Cpm1).

"Nosotros promocionamos de manera recíproca un lenguaje inclusivo que permita evitar discriminaciones, ofendas y modismos que obstaculicen las relaciones de respeto entre todos los integrantes mapuche y no mapuche que conforman la comunidad educativa" (D 3).

Los argumentos dados por la comunidad dejan en evidencia que las prácticas de liderazgo escolar en escuela en contexto mapuche en su mayoría están enlazadas transversalmente con el desarrollo de acciones formadoras que nutren la participación democrática y el trabajo conjunto como espacios que propicien el desarrollo de un lenguaje inclusivo, favoreciendo un clima basado en relaciones de respeto mutuo entre las personas de ascendencia indígena mapuche o no mapuche. En este sentido, Murillo y Krichesky (2015) y Tinajero y Solís (2019) 
y sostienen que construir relaciones de confianza a través de aprendizajes colaborativos asegura buenas prácticas que benefician el compromiso con el desarrollo de una cultura escolar al servicio de una sana convivencia intercultural.

En relación a la dimensión "Desarrollando y gestionando el establecimiento escolar" surgió la quinta categoría:

Categoría 5: Práctica de liderazgo con foco en el reconocimiento del saber mapuche

Los argumentos relacionados a esta categoría contribuyen a expresar que la práctica de liderazgo escolar se concentra en comprender y analizar el contexto mapuche a través de diálogos con la comunidad mapuche y escolar, con el propósito de identificar las reales necesidades de educación a mejorar por la escuela. En relación a lo anterior, se presentan algunas explicaciones que resultaron ser reiterativas, cuando se les pregunta sobre:

¿Cómo se construye una escuela intercultural mapuche?

"Yo, como consejera pedagógica mapuche, consideró que una escuela es intercultural cuando el director conoce, valora y respeta las creencias mapuche y el kimun (saber ancestral)" (Cpm2).

"Para mí como profesora mapuche construir una escuela intercultural requiere de una mirada de educación distinta, centrada en el rakisuam (reflexiones); nosotros hemos tratado como escuela de instaurar en las reuniones de apoderados y profesores esta práctica ancestral porque consideramos que favorece el diálogo compartido y, por sobre todo, aborda las prácticas de escucha atenta que poseían nuestros antepasados para aconsejar o formar a los pichikono (niños)" (D 1).

"Consider que esta escuela es intercultural porque todos los profesores y nuestro director consideran nuestros saberes mapuche, incorporándolos siempre en las clases, porque mi pichikona me pregunta cuando llega a casa e incluso consultan cuando tienen dudas sobre algún conocimiento, siempre nos respeta y se preocupa si mi pichikono (mi niño) no aprende" (Cpm1).

Los argumentos dan cuenta que la práctica de liderazgo pedagógico de escuela en contexto mapuche favorece positivamente su construcción cuando valora, atiende y respeta los saberes culturales y sociales del pueblo mapuche. Asimismo, se destaca el rakizuam como una estrategia transversal de liderazgo intercultural que promociona el desarrollo de prácticas sociales e interpersonales a través del consejo, tiene como propósito según los relatos formar al otro mediante reflexiones críticas de lo que está aprendiendo, va aprender y 
se aprenderá. Al respecto, diversos autores sostienen que transformar el escenario pedagógico con actividades significativas desde el entorno sociocultural y desde una reflexión auténtica gatilla en el aprendiz el interés por aprender (Rojas, 2013; Buitrago et al. 2014; Mellado \& Chaucono, 2016; Arias, 2017; Gutiérrez et al. 2018).Dicho de otra forma, valorar el diálogo y la reflexión como una estrategia de mejoramiento escolar en contexto rural mapuche, transforma la escuela en comunidad de aprendizaje, donde los conocimientos mapuche y escolares se nutren recíprocamente hacia el desarrollo de una formación pertinente y adecuada a la realidad sociocultural del estudiante.

Finalmente, se expone en la Fig. 1 las fases del ciclo del mejoramiento de las prácticas de liderazgo que favorecen el aprendizaje del estudiante, las cuales emergieron desde los registros etnográficos desarrollados en el centro educativo en estudio.

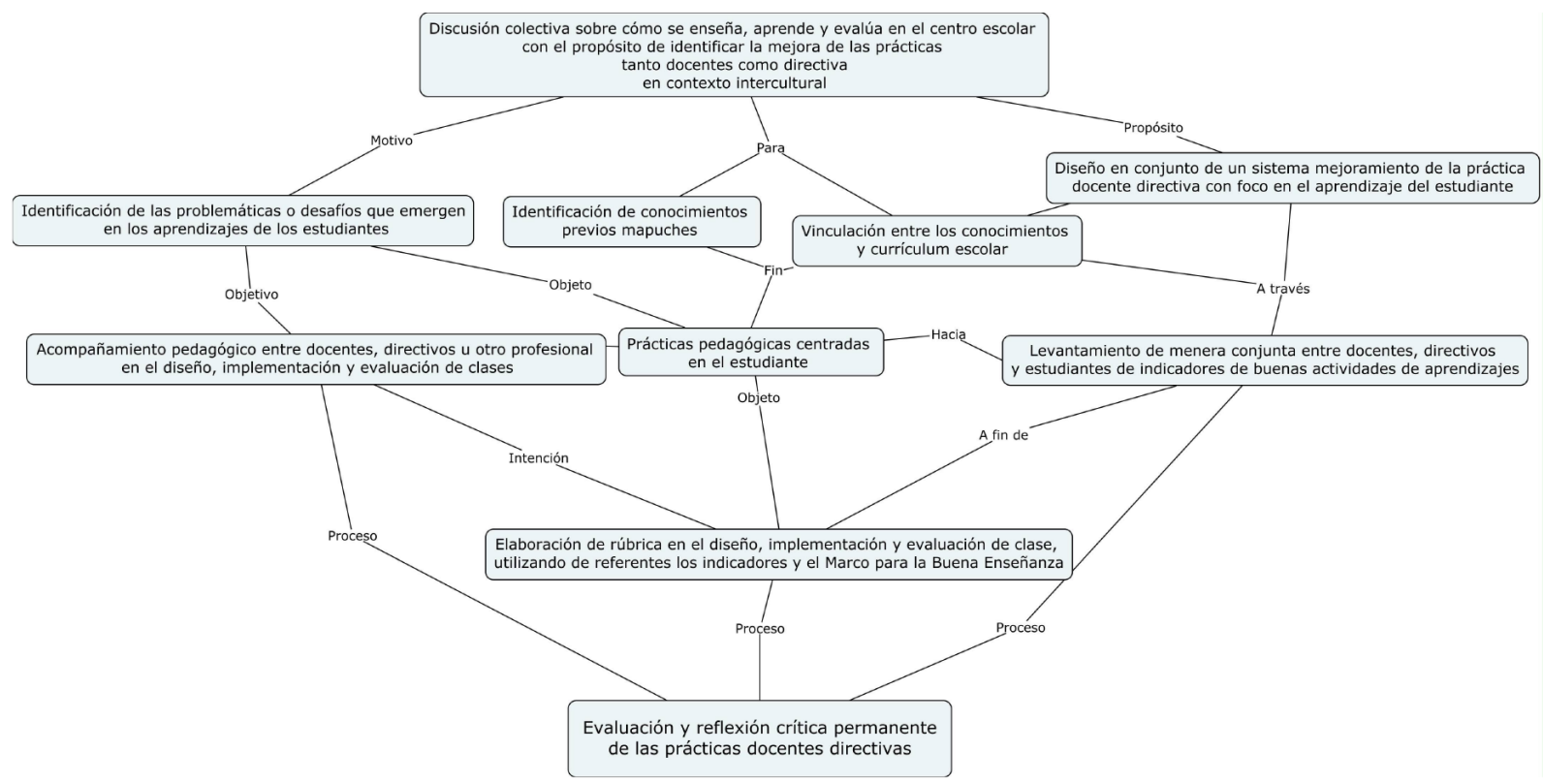

Fig. 1. Fases del ciclo de mejoramiento de prácticas de liderazgo que facilitan el aprendizaje de estudiantes mapuche.

Fuente: Elaboración.

La Fig. 1 muestra el ciclo de mejoramiento de prácticas de liderazgo que facilitan el aprendizaje del estudiante en contexto mapuche, se observa que las fases desarrolladas por la escuela son cíclicas y continuas que requieren de procesos de evaluación y reflexión crítica sobre la práctica de manera permanente. De este modo, la primera fase corresponde a la discusión profesional sobre cómo se enseña, aprende y evalúa en el contexto intercultural, lo que implica que tanto docentes como directivos autoevalúen sus propias prácticas en base a referentes (Marco para la Buena Enseñanza) y agentes (estudiantes, colega par o sabio mapuche) con foco en la mejora de los aprendizajes de los estudiantes. La segunda fase se refiere al diseño conjunto entre docentes y directivos de un sistema de mejoramiento 
de la práctica docente - directiva. De esta manera, el núcleo central de este plan radica en identificar las diversas acciones a realizar por docentes directivos para profundizar en las habilidades, conocimientos y actitudes a mejorar en el estudiantado en relación a sus saberes cotidianos mapuche y conocimientos escolares del currículum prescrito. La tercera fase requiere de un trabajo conjunto entre docentes, directivos y estudiantes para reconocer indicadores de buenas actividades de aprendizajes en base a las experiencias culturales construidas y trayectoria escolar.

La cuarta y quinta fase hace alusión a la elaboración de un instrumento "rúbrica" en la etapa del diseño, implementación y evaluación. En relación al diseño, esta etapa corresponde al trabajo conjunto que realizan docentes, directivo o docente par de las planificaciones de clase, considerando los saberes culturales mapuche, las necesidades e intereses del estudiante e indicadores de buenas prácticas construidas en la fase anterior. La etapa de implementación concierne al proceso del desarrollo de la clase con un docente par o directivo, usando de referente los indicadores emanados en el diseño. La última etapa atañe al proceso evaluativo del diseño e implementación de clase, utiliza los indicadores diseñados en las etapas anteriores siendo un referente guía que orienta las prácticas pedagógicas. La sexta fase pertenece a la identificación de las problemáticas o los desafíos que emergen en los aprendizajes de los estudiantes mapuche, ésta apunta al reconocimiento de los nudos críticos que requieren ser mejorados por medio de las dificultades presentadas en las diversas actividades de aprendizaje o por las autoevaluaciones realizadas a los estudiantes a través de preguntas abiertas que permitan comprender las posibles explicaciones que atribuyen a sus errores o aciertos al construir su aprendizaje desde su conocimiento mapuche.

\section{CONCLUSIONES}

Tras el análisis de los resultados, se pueden extraer algunas conclusiones preliminares que limitan a la comunidad escolar en estudio.

En este caso en particular, las prácticas de liderazgo pedagógico que realizan tanto docentes como directivos ofrecen elementos a considerar para mejorar la gestión y liderazgo de los centros escolares en contexto rural mapuche, entre los cuales se destaca el reconocimiento de los saberes previos mapuche como un conocimiento escolar, lo que evidencia el desarrollo de un currículum pertinente y contextualizado que atiende a los intereses, necesidades y características del territorio local y de las familias mapuche. En la especificidad de las prácticas directivas emergen cinco categorías que parecieran ser fundamentales para asumir un liderazgo que favorezca el aprendizaje del estudiante mapuche.

La primera se refiere a la dimensión "Construyendo e implementando una visión estratégica compartida”. En esta dimensión de la práctica de liderazgo se puede apreciar que involucrar a la comunidad mapuche en las decisiones pedagógicas y directivas favorece la construcción de un enfoque formador desde una mirada intercultural, lo que permite 
desarrollar actividades de aprendizajes más pertinentes y significativas para el estudiante. Lo anterior, se traduce en el compromiso e identidad cultural que logra construir la familia mapuche con el centro escolar en estudio.

Respecto a la dimensión "Desarrollando las capacidades profesionales", se puede constatar que la reflexión crítica de la práctica que realizan tanto docentes como directivos se aborda de manera transversal a través de las discusiones profesionales que surgen en base a las necesidades y desafíos focalizados recurrentemente en mejorar la calidad de las actividades de aprendizajes en virtud de los conocimientos cotidianos mapuche que presentan los estudiantes. Esta situación nutre el desarrollo de una cultura escolar centrada en un trabajo colaborativo, en decisiones compartidas y acciones que ayudan a mejorar la pertinencia de la enseñanza para el desarrollo de una educación intercultural.

En relación a la tercera dimensión, "Liderando los procesos de enseñanza y aprendizaje", se visualiza que la práctica de liderazgo está focalizada en asegurar que el estudiante aprenda en los diversos contextos educativos, donde se destaca el análisis riguroso que realizan tanto docentes como directivo de los aprendizajes previos mapuche construidos por los niños y niñas a través de la interacción recíproca con el medio natural y cultural. En este sentido, se pueden identificar tempranamente desde el currículum escolar los conocimientos, habilidades y actitudes aprendidas por los estudiantes antes de iniciar una unidad de aprendizaje. Asimismo, se evidencia que el mapeo curricular diseñado por el profesorado y directivo facilita la organización de las aulas multigrado en relación a los objetivos de aprendizaje que se deben aprender, según nivel y asignatura; a su vez, permite diseñar desde los intereses del estudiante proyectos de aulas más contextualizados a su realidad sociocultural. El hallazgo anterior evidencia la existencia de una comprensión profunda por parte de docentes y directivos de los saberes pedagógicos, disciplinarios y culturales que se requieren profundizar o mejorar en los estudiantes del curso o nivel que atienden, demostrando que la práctica que realiza el directivo influye positivamente en la calidad de la tarea.

En la dimensión "Gestionando la convivencia y la participación de la comunidad escolar" se puede documentar que la práctica de liderazgo que propicia el aprendizaje integral de todos se aborda transversalmente en la comunidad escolar, lo que trae consigo el desarrollo de una cultura que promociona relaciones de confianza, respeto y colaboración mutua entre todos los integrantes que conforman la comunidad. De manera específica, se puede sostener que esta práctica se cruza con los lineamientos que persiguen las políticas públicas en Chile sobre el derecho de ser respetados y tratados con dignidad desde un marco de inclusión e interculturalidad.

Respecto a la dimensión "Desarrollando y gestionando el establecimiento escolar" se testea que la práctica de liderazgo con foco en el reconocimiento del saber mapuche mejora los aprendizajes porque sitúa al estudiante como protagonista activo, garantizando escenarios interculturales auténticos y significativos para aprender. De esta forma, los conocimientos mapuche y escolares se interrelacionan mutuamente para alcanzar construcciones colectivas 
en la toma de decisiones que apunten al crecimiento, desarrollo y transformación de una educación que garantice la equidad de aprendizaje tanto en niños mapuche como no mapuche.

Los hallazgos encontrados en este estudio permiten identificar las particularidades y singularidades que presenta el liderazgo escolar en contexto rural mapuche, lo cual se constata cuando se aborda de manera comunitaria y participativa la construcción de un enfoque de escuela intercultural, permitiendo de este modo desarrollar comunidades de aprendizajes más inclusivas e interculturales. Asimismo, se evidencia que comprender la cosmovisión de nuestro pueblo mapuche facilita el diálogo recíproco y formador entre ambas culturas. Cabe destacar que el Marco para la Buena Dirección y Liderazgo Escolar (2015) contribuyó a fortalecer de manera colaborativa prácticas de mejoramiento de manera ascendente, en las cuales la participación activa y el involucramiento son decisivos, y éstas no dependen del contexto sociocultural, sino del desarrollo de un liderazgo compartido, de oportunidades para aprender colaborativamente, de decisiones colectivas y de responsabilidades mutuas entre todos los integrantes que conforman la comunidad educativa.

Evidentemente, el estudio presenta como principal limitación el muestreo, el cual puede ser ampliado para generalizar los resultados presentes en este estudio de caso. De este modo, también se recomienda realizar investigaciones desde una metodología mixta, esto con la finalidad de contribuir al desarrollo de evidencias estadísticamente significativas.

\section{REFERENCIAS}

Arias, J. (2017). Problemas y retos de la educación rural en Colombia. Revista educación y ciudad, 33, 53-62.

Bellei, C., Valenzuela, J. P., Vanni, X., \& Contreras, D. (2014). Lo aprendí en la escuela. ¿Cómo se logran procesos de mejoramiento escolar? Santiago de Chile: LOM.

Beltrán, J., \& Osses, S. (2018). Transposición didáctica de saberes culturales mapuche en escuelas situadas en contextos interculturales. Revista Latinoamericana de Ciencias Sociales, Niñez y Juventud, 16(2), 669-684.

Beltrán, J., \& Pérez, S. (2018). Factores que dificultan la relación educativa entre la educación escolar y el saber y conocimiento mapuche. Revista Diálogo Andino, 57, 9-20.

Bender, P. (2017). Lo que deben hacer los líderes para construir una mejor escuela. En J. Weinstein \& G. Muñoz (Eds.), Mejoramiento y Liderazgo en la Escuela: Once Miradas (pp. 113-149). Santiago de Chile: Ediciones Universidad Diego Portales.

Bolívar, A. (2015). Evaluar el liderazgo pedagógico de la dirección escolar. Revisión de enfoques e instrumentos. Revista Iberoamericana de Evaluación Educativa, 8(2), 15-39.

Bolívar, A., Caballero, K., \& García, M. (2017). Evaluación multidimensional del liderazgo pedagógico: claves para la mejora escolar. Ensaio: Avaliação e Políticas Públicas em Educação, 25(95), 483-506.

Buitrago, M., Baptista, E., \& Chiquinquirá, L. (2014). El liderazgo wayuu: una perspectiva 
indígena del gerente público venezolano. Revista Especializada en Periodismo y Comunicación, 43(1), 60-74.

Chaucono, J., Mellado, M., \& Yuste, R. (2017). Creencias de directivos escolares sobre educación intercultural de escuelas rurales en contexto mapuche. file://C:/Users/ beltr/Downloads/031_Chaucono.pdf (22 de enero de 2018)

Day, C., Gu, Q., \& Sammons, P. (2016). The impact of leadership on student outcomes: How successful school leaders use transformational and instructional strategies to make a difference. Educational Administration Quarterly, 52(2), 221-258.

Fullan, M. (2017). Liderar los aprendizajes: acciones concretas en pos de la mejora escolar. En J. Weinstein \& G. Muñoz (Eds.), Mejoramiento y Liderazgo en la Escuela: Once Miradas (pp. 182-192). Santiago de Chile: Ediciones Universidad Diego Portales.

Guba, E., \& Lincoln, Y. (2012). Controversias paradigmáticas, contradicciones y confluencias emergentes. En Denzin, N \& Lincoln, Y (Eds.),. Manual de metodología cualitativa vol. II. Paradigmas y perspectivas en disputa. España: Gedisa editorial.

Gurdián, A. (2007). El paradigma cualitativo en la investigación socioeducativa. Print Center, San José, Costa Rica.

Gutiérrez, M., Martín, V., \& Jenaro, C. (2018). La cultura, pieza clave para avanzar en la inclusión en los centros educativos. Revista de Educación Inclusiva, 11(2), 13-26.

Lara, A. (2014). Aprender a leer y escribir en lengua Mapudungun, como elemento de recuperación y promoción de la cultura mapuche en la sociedad del siglo XXI (Tesis doctoral). Universidad Autónoma de Barcelona, España. Disponible en: https://www. tdx.cat/bitstream/handle/10803/107892/milm1de1.pdf?sequence=1

Leithwood, K. (2011). The fourth essential components of the leader's repertoire. En K. Leithwood \& K. S. Louis (Eds.). Linking leadership to student learning (pp. 57-67). San Francisco: Jossey-Bass.

Leithwood, K., Day, C., Sammons, P., Harris, A., \& Hopkins, D. (2006). Successful school leadership. What it is and how it influences pupil learning: National College for School Leadership.Nottingham: DFES Publicaciones,

Mellado, M., \& Huaiquimil, A. (2015). An Intercultural Education: Teaching Reading in a Mapuche Context. Revisit International Education Studies, 8(10), 28-39.

Mellado, M., \& Chaucono, J. (2016). Liderazgo pedagógico para reestructurar creencias docentes y mejorar prácticas de aula en contexto mapuche. Revista Electrónica Educare, 20(1), 1-18.

Mellado, M., Chaucono, J., \& Villagra, C. (2017). Creencias de directivos escolares; implicancias en el liderazgo pedagógico. Revista Psicología Escolar e Educacional, 21(3), 541-548.

Ministerio de Educación de Chile, (2015). Marco para la Buena Dirección y el Liderazgo Escolar. Santiago de Chile: Editora e Imprenta Maval Ltda.

Murillo, F., \& Krichesky, G. (2015). Mejora de la Escuela: Medio siglo de lecciones aprendidas. REICE. Revista Iberoamericana sobre Calidad, Eficacia y Cambio en Educación, 13(1), 
69-102.

Quintriqueo, S., \& Torres, H. (2013). Construcción de conocimiento mapuche y su relación con el conocimiento escolar. Estudios Pedagógicos, 39(1), 199-216.

Quintriqueo, S., Morales, S., Quilaqueo, D., \& Arias, K. (2016). Interculturalidad para la formación inicial docente: desafíos para construir un diálogo intercultural. Temuco, Chile: Ediciones Universidad Católica de Temuco.

Rincón - Gallardo, S. (2019). Liberating Learning: Educational Change as Social Movement. Routledge.

Robinson, V., Lloyd, C., \& Rowe, K.J. (2014). El impacto del liderazgo en los resultados de los estudiantes. REICE. Revista Iberoamericana sobre Calidad, Eficacia y Cambio en Educación, 12(4), 12-40.

Rojas, R. (2013). El liderazgo comunitario y su importancia en la intervención comunitaria. Psicología para América Latina, 57-76.

Sammons, P., Gu, Q., Day, C., \& Ko, J. (2011). Exploring the impact of school leadership on pupil outcomes: Results from a study of academically improved and effective schools in England. International journal of educational management, 25(1), 83-101.

Tinajero, M., \& Solís, S. (2019). Inclusión y gestión escolar en escuelas indígenas de México. Revista Perspectiva Educacional, 58(2), 147-168.

Vaillant, D., \& Marcelo, C. (2015). El ABC y D de la formación docente. Madrid: Editorial Narcea. 174 páginas. ISBN: 978-84-277-2085-5 D.L.: M-5805-2015.

Weinstein, J., \& Muñoz, G. (Eds.). (2017). Mejoramiento y Liderazgo en la Escuela: Once Miradas. Santiago de Chile: Ediciones Universidad Diego Portales. 